\title{
The Effect of an Automated Clinical Reminder on Weight Loss in Primary Care
}

\section{Jason S. O'Grady, MD, Tom D. Thacher, MD, and Rajeev Chaudhry, MBBS, MPH}

Background: Overweight and obese individuals have increased health risks. Clinical reminders positively affect health outcomes in diabetes and osteoporosis, but the effect of automated prompts on weight loss in obesity has not been studied. Our objective was to determine whether an automatic prompt for the clinician to recommend lifestyle changes to patients with a body mass index (BMI) $>25 \mathrm{~kg} / \mathrm{m}^{2}$ led to greater weight loss over a 3- to 6-month interval compared with the absence of a clinical reminder.

Methods: We conducted a retrospective analysis of electronic medical records of obese adult patients with a BMI $>25 \mathrm{~kg} / \mathrm{m}^{2}$ who were seen in 2009 and 2010 , before and after implementation of an automated printed clinical reminder, respectively. We evaluated 1600 patients in each of the control and intervention groups. The primary outcome was the mean change in BMI between the control and intervention groups. Multiple linear regression was used to assess the effect of the clinical reminder on the change in BMI while adjusting for baseline BMI and potential confounding factors.

Results: The reduction in BMI (mean \pm standard deviation) in the group with the clinical reminder $\left(-0.084 \pm 1.56 \mathrm{~kg} / \mathrm{m}^{2}\right)$ was not significantly greater than the control group $\left(-0.053 \pm 1.49 \mathrm{~kg} / \mathrm{m}^{2}\right.$; $P=.56)$. A regression model incorporating the clinical reminder, age, baseline BMI, obesity diagnosis, diabetes, and hyperlipidemia found that baseline BMI $(P<.001)$, obesity diagnosis $(P<.001)$, age $(P=.001)$, and hyperlipidemia diagnosis $(P=.02)$ were significant predictors of weight loss, but the clinical reminder was not $(P=.78)$. There was a significant interaction between the clinical reminder and baseline BMI $(P=.005)$, as the prompt increased weight loss more in those with lower baseline BMI.

Conclusion: Automated clinical reminders alone do not improve weight loss in overweight and obese patients. Physician diagnoses of obesity or hyperlipidemia were associated with weight loss, suggesting that formally noting these diagnoses contributes to successful weight loss. (J Am Board Fam Med 2013;26: 745-750.)

Keywords: Electronic Medical Records, Obesity, Practice Management

Overweight and obese individuals, defined as a body mass index (BMI) $>25 \mathrm{~kg} / \mathrm{m}^{2}$, are at an increased risk

This article was externally peer reviewed.

Submitted 17 December 2012; revised 13 May 2013; accepted 28 May 2013.

From the Department of Family Medicine (JSO, TDT) and the Division of Primary Care, Department of Internal Medicine (RC), Mayo Clinic, Rochester, MN.

Funding: A small grant was obtained from the Mayo Clinic Family Medicine Research Committee to offset the cost of data abstraction.

Conflict of interest: $\mathrm{RC}$ is an employee of the Mayo Clinic and the inventor of the GDMS software referenced in this publication. Mayo Clinic has licensed this technology to a commercial entity (VitalHealth Software) but to date has received no royalties. $\mathrm{RC}$ receives no royalties from the licensing of this technology.

Corresponding author: Jason S. O'Grady, MD, Department of Family Medicine, Mayo Clinic, 200 First St. SW, Rochester, MN 55905 (E-mail: ogrady.jason@mayo.edu). for a multitude of diseases, including diabetes, coronary artery disease, stroke, hypertension, hypercholesterolemia, sleep apnea, and osteoarthritis. Obesity and weight gain have been implicated in approximately $20 \%$ of all cancer cases, including cancer of the thyroid, colon, breast, prostate, and kidneys. ${ }^{1}$

Obesity has become a global pandemic. ${ }^{2}$ The Centers for Disease Control and Prevention estimates that approximately 112,000 obesity-associated deaths occur annually in the United States alone. ${ }^{3}$ As of 2008 , the annual cost of obesity in America was $\$ 147$ billion. ${ }^{4}$ Despite efforts to slow or reverse populationwide weight gain, obesity rates continue to increase. More than one-third (35.7\%) of Americans currently have a $\mathrm{BMI}>30 \mathrm{~kg} / \mathrm{m}^{2}{ }^{5}$. While physicians underdiagnose and undertreat obesity in their patients, ${ }^{6}$ life- 
style modification has been shown to achieve modest long-term weight loss. ${ }^{7}$

Over the past few years, the US Department of Health and Human Services has placed increased emphasis on the meaningful use of electronic medical records. ${ }^{8}$ Clinical reminders have been shown to positively affect the health outcomes of several health conditions. Breast cancer screening rates were significantly better when using an automated clinical prompt. ${ }^{9}$ Similar improvement in screening for abdominal aortic aneurysms was noted using programmed reminders. ${ }^{10}$ Clinical reminders positively affected osteoporosis screening rates. ${ }^{11}$

Beneficial effects of automated clinical reminders were not limited to preventive screening. A small retrospective analysis of patients with diabetes mellitus at the Mayo Clinic found that automated prompts significantly improved low-density lipoprotein cholesterol levels, and a trend toward improved glycosylated hemoglobin values was observed. ${ }^{12}$ Clinical reminders improve the completion of asthma assessments, even during clinic visits for non-asthma-related issues. ${ }^{13}$ Despite these advances in health information technology, no study has reported the effect of automated clinical reminders on obesity. We hypothesized that automated clinical reminders to physicians to recommend lifestyle changes to patients with a BMI $>25$ $\mathrm{kg} / \mathrm{m}^{2}$ would lead to a greater decrease in BMI over 3 to 6 months compared with the absence of a clinical reminder.

\section{Methods}

More than 140,000 patients are seen annually for primary care at the Employee and Community Health (ECH) practice of the Mayo Clinic in Rochester, Minnesota. The Generic Disease Management System (GDMS) is a web-based application created by VitalHealth, a partnership between the Mayo Clinic and the Noaber Foundation. The GDMS extracts information from the electronic medical record to improve patient care with rulesbased reminders for preventative services, diabetes, and coronary artery disease care. ${ }^{9}$ The application is frequently revised to include updated recommendations. In the ECH practice, a summary of GDMS recommendations is printed at each patient visit to enable the clinician to discuss recommendations with the patient. In January 2010, an automated clinical reminder for the clinician to recom- mend lifestyle modification for all adult patients with a BMI $>25 \mathrm{~kg} / \mathrm{m}^{2}$ was added to the GDMS (Figure 1). A printed copy of the recommendations, including this reminder, was given to the patient.

An independent data abstractor retrospectively extracted data from the records of patients with a $\mathrm{BMI}>25 \mathrm{~kg} / \mathrm{m}^{2}$ who presented for health maintenance visits between January and April 2010 and who had repeat visits for any reason between July and October 2010. All adults aged 18 to 75 years seen in the $\mathrm{ECH}$ practice with consent for medical records research and a BMI $>25 \mathrm{~kg} / \mathrm{m}^{2}$ were eligible for inclusion. In a prior study of Mayo Clinic patients residing within the $\mathrm{ECH}$ catchment area, $5.8 \%$ of patients did not consent to medical records research. ${ }^{14}$ To maximize the generalizability of the results, no exclusion criteria were used. The change in BMI was compared with the change in BMI of a historical control group of patients with $\mathrm{BMI}>25$ $\mathrm{kg} / \mathrm{m}^{2}$ seen in the ECH practice during the previous year (between January and April 2009) and who had repeat visits between July and October 2009, before the automated clinical reminder was instituted. Other data abstracted from the electronic medical records included patient sex, age, associated diagnoses (diabetes, hyperlipidemia, concurrent psychiatric illness), tobacco use, provider sex, provider affiliation (internal medicine vs. family medicine), clinic location, visit dates, and obesity diagnoses noted in the record.

$A t$ test was used to compare the mean change in BMI between the control and study groups, our primary outcome of interest. Multiple linear regression analysis was used to assess the effect of the clinical reminder on the change in BMI while adjusting for baseline BMI and potential confounding variables. Confounding variables were selected based on consideration of their potential to alter the degree of weight loss observed or the likelihood of responding to the prompt. Interaction terms were included to assess whether the response to the clinical prompt differed between subgroups of patients. Potential interactions with the BMI prompt were explored for all confounding variables. $P<$ .05 was considered significant. A sample size of 1600 patients in each group was chosen to provide $80 \%$ power to detect a difference in BMI change of 0.5 between the 2 groups with $95 \%$ confidence, ${ }^{15}$ assuming a standard deviation in BMI of $5.0 \mathrm{~kg} / \mathrm{m}^{2}$. All statistical analyses were performed using JMP 9.0.1 (SAS Institute, Inc., Cary, NC). 
Figure 1. Generic Disease Management System output with obesity prompt highlighted.

Mayo Clinic - GDMS for decision support

Page 1 of 1

Mayo Clinic - GDMS

Last name:
First name:
Clinic Number:
DOB:
Physician:

(C) Recommendation For You

Provide tobacco cessation advice Advise lifestyle counseling as $\mathrm{BMI}>25$.

90 Recommendations in 90 Days

7. Preventive Services \& Other Tests Done

Influenza vaccine 27-OCT-2011

Tetanus vaccine 13-MAY-2010

Pneumococcal vaccine 26-JAN-2008

AAA screening 30-NOV-2000

Colon X-ray 16-OCT-2002

Mammogram 08-JUN-2011

Bone density screening 14-NOV-2005

Colonoscopy 24-JAN-2008

Echocardiogram 17-OCT-2011

Electrocardiogram 21-OCT-2011

\section{f Vital Signs and Conditions}

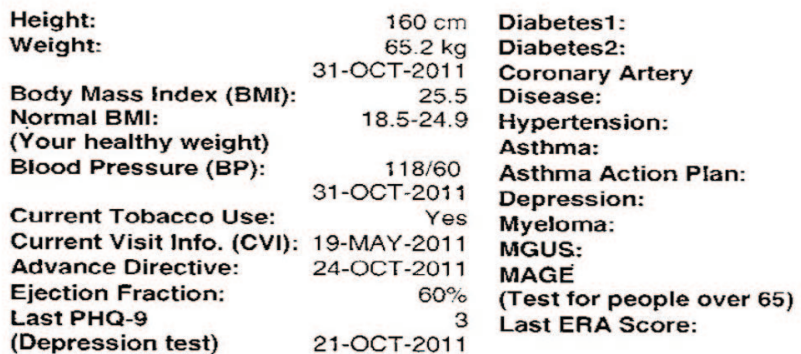

\section{Labs Results}

denotes abnormal results

TEST (your normal range)

Recent value

Previous value

HEMOGLOBIN $(12.0-15.5 \mathrm{~g} / \mathrm{dL})$

Protein in blood that carries oxygen

SODIUM (135-145 mmol/L)

POTASSIUM (3.6-5.2 mmol/L)

A salt in the blood

CREATININE (0.6-1.1 mg/dL)

Measures kiơney function

EGFR (>60)

Mcasuros kidncy function

GLUCOSE (70-100 mg/dL)

Measures sugar in the blood

RANDOM MICROALB.

Protein in the urine

TRIGLYCERIDES

Fats from oxcossive calorics

HDL

Good Cholestero

LDL

Bad Cholosterol

TOTAL CHOLESTEROL

Includes triglycenides. HDL \& LDL

AST-SGOT (8-43 U/L)

Enzyme tound in liver colls

ALT-SGPT

Enzyme found in liver cells

TSH $(0.3-5.0 \mathrm{mIU} / \mathrm{L})$

Thyroid function lest

INR $(0.9-1.3)$

Routine warfarin monitoring
Data were evaluated for obvious outliers, deemed as a BMI change greater than $\pm 15 \mathrm{~kg} / \mathrm{m}^{2}$. These 9 patients' records were reviewed for possible errors in entry. Height and weight data were corrected when clear typographical errors were made. The 2 patients for whom correct heights and weights could not be confirmed were excluded.

\section{Results}

Characteristics of the study and control subjects were similar (Table 1). The mean \pm standard deviation change in weight was $-0.51 \pm 9.83 \mathrm{~kg}$ in the clinical reminder group, which did not significantly differ from the $-0.35 \pm 9.40-\mathrm{kg}$ change in the control group $(P=.64)$. Similarly, the reduction in BMI was not significantly greater in the group with the clinical reminder $(-0.084 \pm$ $\left.1.56 \mathrm{~kg} / \mathrm{m}^{2}\right)$ compared with the control group $\left(-0.053 \pm 1.49 \mathrm{~kg} / \mathrm{m}^{2} ; P=.56\right)$. A subgroup analysis of the 1830 obese subjects did not demonstrate a significant difference in the reduction in BMI between the groups with $(-0.13 \pm 1.74$ $\left.\mathrm{kg} / \mathrm{m}^{2}\right)$ and without $\left(-0.18 \pm 1.69 \mathrm{~kg} / \mathrm{m}^{2}\right)$ the clinical reminder $(P=.50)$. Including the 2 data outliers in the analysis did not change the study conclusions.

A linear regression model incorporating the clinical reminder, age, baseline BMI, obesity diagnosis, diabetes, and hyperlipidemia found that baseline BMI $(P<.001)$, obesity diagnosis $(P<$ 
Table 1. Characteristics of Study Participants

$(\mathrm{n}=1600)$

\begin{tabular}{|c|c|c|}
\hline Characteristics & $\begin{array}{c}\text { No } \\
\text { Prompt }\end{array}$ & Prompt \\
\hline \multicolumn{3}{|l|}{ Sex } \\
\hline Male & $634(39.6)$ & $659(41.2)$ \\
\hline Female & $966(60.4)$ & $941(58.8)$ \\
\hline \multicolumn{3}{|l|}{ Age (years) } \\
\hline $18-29$ & $168(10.5)$ & $113(7.1)$ \\
\hline $30-44$ & $289(18.1)$ & $273(17.1)$ \\
\hline $45-59$ & $611(38.2)$ & $660(41.2)$ \\
\hline $60-75$ & $532(33.2)$ & $554(34.6)$ \\
\hline \multicolumn{3}{|l|}{ Smoking } \\
\hline Yes & $156(9.8)$ & $157(9.8)$ \\
\hline No & $1344(84)$ & $1345(84)$ \\
\hline Unknown & $100(6.2)$ & $98(6.2)$ \\
\hline \multicolumn{3}{|l|}{ Diabetes } \\
\hline Yes & $299(18.7)$ & $310(19.4)$ \\
\hline No & $1301(81.3)$ & $1290(80.6)$ \\
\hline \multicolumn{3}{|l|}{ Hyperlipidemia } \\
\hline Yes & $713(44.6)$ & $720(45)$ \\
\hline No & $887(55.4)$ & $880(55)$ \\
\hline \multicolumn{3}{|l|}{ Obesity diagnosis } \\
\hline Yes & $185(11.6)$ & $198(12.4)$ \\
\hline No & $1415(88.4)$ & $1402(87.6)$ \\
\hline \multicolumn{3}{|l|}{ Psychiatric illness } \\
\hline Yes & $89(5.6)$ & $100(6.3)$ \\
\hline No & $1511(94.4)$ & $1500(93.7)$ \\
\hline \multicolumn{3}{|l|}{ Baseline BMI $\left(\mathrm{kg} / \mathrm{m}^{2}\right)$} \\
\hline Mean \pm SD & $32.3 \pm 7.4$ & $32.6 \pm 7.2$ \\
\hline $25.0-29.9$ & $706(44.1)$ & $663(41.4)$ \\
\hline $30.0-34.9$ & $476(29.8)$ & $498(31.1)$ \\
\hline$\geq 35.0$ & $417(26.1)$ & $439(27.4)$ \\
\hline Time interval (mean days $\pm \mathrm{SD}$ )* & $203 \pm 44$ & $205 \pm 42$ \\
\hline
\end{tabular}

Data are n (\%) unless otherwise indicated.

*Between initial and follow-up visits.

BMI, body mass index; SD, standard deviation.

$.001)$, age $(P=.001)$, and hyperlipidemia diagnosis $(P=.02)$ were significant predictors of weight loss, but the clinical reminder was not (Table 2). There was a significant interaction between the clinical reminder and baseline BMI $(P=.005)$ because the prompt increased weight loss more in those with a lower baseline BMI. The proportion of individuals achieving $>5 \%$ weight loss (a degree of weight loss associated with a reduced risk of type 2 diabetes) was not significantly greater in the group with the clinical reminder $(10.8 \%)$ than in the control group $(9.2 \% ; P=.08)$.

\section{Discussion}

While failing to show the effectiveness of clinical reminders on obesity, this study does reinforce the widely held notion that the effective management of obesity remains difficult and requires a multidisciplinary approach. ${ }^{16}$ Despite proven benefits of automated clinical reminders in asthma, osteoporosis screening, mammography, and diabetic control, automatic prompts alone did not improve weight loss in overweight and obese patients. Obesity is caused by multiple physical, psychological, social, and societal factors. A clinical reminder may serve as a starting point for discussion of obesity treatment, but it cannot get to the root causes of obesity in the individual patient.

A number of factors could have contributed to the failure of clinical prompts to affect obesity. With the influx of a myriad of electronic reminders, clinicians may have simply succumbed to "alert fatigue" and ignored this reminder. ${ }^{17}$ In addition, a published cross-sectional analysis has shown that physicians spend an average of 16.3 minutes for each patient visit. ${ }^{18}$ Clinicians simply may not have had time to address obesity management during the allotted appointment time. Last, a number of barriers to the effective treatment of obesity in the primary care setting exist, such as stigma, cost, patient's previous experience with weight loss, physician's unwillingness to take responsibility for obe-

Table 2. Results of Multiple Linear Regression Analysis of Factors Associated With a Change in Body Mass Index (BMI)

\begin{tabular}{lccc}
\hline Variables & Effect Coefficient & $95 \%$ Confidence Interval & $P$ Value \\
\hline Clinical reminder & -0.0074 & -0.060 to 0.045 & .78 \\
Baseline BMI $\left(\mathrm{kg} / \mathrm{m}^{2}\right)$ & -0.014 & -0.022 to -0.0062 & $.0004^{*}$ \\
Diabetes diagnosis & -0.017 & -0.087 to 0.054 & .64 \\
Hyperlipidemia diagnosis & -0.069 & -0.13 to -0.011 & $.02^{*}$ \\
Obesity diagnosis & -0.20 & -0.28 to -0.11 & $<.0001^{*}$ \\
Age (years) & -0.0069 & -0.011 to -0.0027 & $.0012^{*}$ \\
\hline
\end{tabular}

${ }^{*}$ Statistically significant, $P<.05$. 
sity management, and perceived limited skill in treating obesity. ${ }^{19}$

Change in health care delivery from our current fee-for-service model to include more population management by primary care physicians could improve reimbursement for the management of chronic diseases such as obesity and provide an impetus for increased physician responsibility for patient outcomes such as weight loss. ${ }^{20}$ Furthermore, care managers have been shown to improve the management of chronic illnesses such as diabetes. ${ }^{21}$ Further study is needed to show whether care managers have an affect on obesity. If so, automated clinical reminders to the care manager may improve weight loss.

In our study, physician diagnoses of obesity or hyperlipidemia were associated with significant weight loss, suggesting that formally noting these diagnoses in the chart contributes to successful weight loss in patients. An alternative explanation for this association is that physicians are more likely to record a diagnosis of obesity or hyperlipidemia in patients who are actively working on losing weight. Inclusion of the diagnosis of obesity in the patient's chart may indicate the importance given by the physician to addressing obesity. We were unable to assess whether discussion of obesity with the patient actually occurred in response to the clinical reminder, but it has been shown that physicians have less emotional rapport with their obese patients in comparison to their normal-weight patients. ${ }^{22}$ WeightWatchers was significantly better and more cost-effective at achieving weight loss after 1 year than primary care programs directed by well-trained clinicians and ancillary staff. ${ }^{23}$

Our study did have several limitations. Regardless of the use of multiple regression analysis, unmeasured residual confounders could have attenuated the effect of clinical reminders. Coinciding with the release of the clinical reminder for obesity, patients in 2010 had access to a wellness consult at a fitness center associated with the Mayo Clinic. However, relatively few patients took advantage of this opportunity, which would have been expected to be associated with a greater effect of the clinical reminder. Second, during the study interval we could not control for the addition of clinical care managers who were involved in managing patients with diabetes, who accounted for approximately $20 \%$ of the overweight and obese population of patients. Last, the entire format of the GDMS report changed when the clinical reminder for obesity was added.

\section{Conclusions}

Obesity will be a growing burden for our patients and the health system overall. Clinical reminders alone are not enough to equip physicians to effectively address obesity in primary care. More research is undoubtedly needed to discover the most effective ways of turning the tide in this obesity epidemic. Family physicians are uniquely positioned to address the treatment of obesity by fully understanding all the associated environmental, social, and economic pressures on obese patients. Only through properly educating patients on the devastating health effects of obesity, partnering with them to achieve meaningful weight loss, and investigating novel methods of achieving and maintaining weight loss can we hope to stem the tide.

The authors are grateful to Mrs. Julie Maxson for her assistance with data abstraction.

\section{References}

1. Wolin KY, Carson K, Colditz GA. Obesity and cancer. Oncologist 2010;15:556-65.

2. Swinburn BA, Sacks G, Hall KD, et al. The global obesity pandemic: shaped by global drivers and local environments. Lancet 2011;378:804-14.

3. Flegal KM, Graubard BI, Williamson DF, Gail MH. Excess deaths associated with underweight, overweight, and obesity. JAMA 2005;293:1861-7.

4. Finkelstein EA, Trogdon JG, Cohen JW, Dietz W. Annual medical spending attributable to obesity: payer-and service-specific estimates. Health Aff (Millwood) 2009;28:w822-31.

5. Ogden CL, Carroll MD, Kit BK, Flegal KM. Prevalence of obesity in the United States, 2009-2010. NCHS Data Brief 2012;(82):1-8.

6. Ma J, Xiao L, Stafford RS. Underdiagnosis of obesity in adults in US outpatient settings. Arch Intern Med 2009;169:313-4.

7. Unick JL, Beavers D, Bond DS, et al. The long-term effectiveness of a lifestyle intervention in severely obese individuals. Am J Med 2013;126:236-42, 242.e1-2.

8. Blumenthal D, Tavenner M. The "meaningful use" regulation for electronic health records. $\mathrm{N}$ Engl J Med 2010;363:501-4.

9. Chaudhry R, Scheitel SM, McMurtry EK, et al. Web-based proactive system to improve breast cancer screening: a randomized controlled trial. Arch Intern Med 2007;167:606-11. 
10. Chaudhry R, Tulledge-Scheitel SM, Parks DA, et al. Use of a web-based clinical decision support system to improve abdominal aortic aneurysm screening in a primary care practice. J Eval Clin Pract 2012;18:666-70.

11. DeJesus RS, Angstman KB, Kesman R, et al. Use of a clinical decision support system to increase osteoporosis screening. J Eval Clin Pract 2012;18:89-92.

12. Chaudhry R, Tulledge-Scheitel SM, Thomas MR, et al. Clinical informatics to improve quality of care: a population-based system for patients with diabetes mellitus. Inform Prim Care 2009;17:95-102.

13. Lim KG, Rank MA, Cabanela RL, et al. The asthma ePrompt: a novel electronic solution for chronic disease management. J Asthma 2012;49:213-8.

14. Jacobsen SJ, Xia Z, Campion ME, et al. Potential effect of authorization bias on medical record research. Mayo Clin Proc 1999;74:330-8.

15. Bazzano AT, Zeldin AS, Diab IR, et al. The Healthy Lifestyle Change Program: a pilot of a communitybased health promotion intervention for adults with developmental disabilities. Am J Prev Med 2009;37 (6 Suppl 1):S201-8.

16. Cerezo C, Segura J, Praga M, Ruilope LM. Guidelines updates in the treatment of obesity or metabolic syndrome and hypertension. Curr Hypertens Rep 2013;15:196-203.
17. Peterson JF, Bates DW. Preventable medication errors: identifying and eliminating serious drug interactions. J Am Pharm Assoc (Wash) 2001;41:159-60.

18. Blumenthal D, Causino N, Chang YC, et al. The duration of ambulatory visits to physicians. J Fam Pract 1999;48:264-71.

19. Gunther S, Guo F, Sinfield P, et al. Barriers and enablers to managing obesity in general practice: a practical approach for use in implementation activities. Qual Prim Care 2012;20:93-103.

20. Hutchison B, Glazier R. Ontario's primary care reforms have transformed the local care landscape, but a plan is needed for ongoing improvement. Health Aff (Millwood) 2013;32:695-703.

21. Hiss RG, Armbruster BA, Gillard ML, McClure LA. Nurse care manager collaboration with communitybased physicians providing diabetes care: a randomized controlled trial. Diabetes Educ 2007;33:493-502.

22. Gudzune KA, Catherine Beach M, Roter DL, Cooper LA. Physicians build less rapport with obese patients. Obesity (Silver Spring) 2013 March 20. [Epub ahead of print].

23. Jolly K, Lewis A, Beach J, et al. Comparison of range of commercial or primary care led weight reduction programmes with minimal intervention control for weight loss in obesity: lighten Up randomised controlled trial. BMJ 2011;343:d6500. 